Original Article

\title{
Ultrasound measurements of the median nerve at the carpal tunnel in ambulant chronic stroke patients: comparison between paretic and non-paretic sides
}

\author{
Mitsutoshi Shinoda, RPT ${ }^{1)}$, Motoyuki Abe, MD, $\mathrm{PhD}^{2)^{*}}$ \\ 1) Yoshida Orthopedic Hospital: 7-100 Mitachi-cho, Toyota-shi, Aichi 471-0811, Japan \\ 2) Faculty of Care and Rehabilitation, Seijoh University, Japan
}

\begin{abstract}
Purpose] The purpose of this study was to examine the cross-sectional area (CSA) and longitudinal sliding length (LSL) of the median nerve bilaterally in patients with ambulant chronic hemiplegia and to compare these measurements with those in healthy controls using ultrasound. [Participants and Methods] Forty patients with hemiplegia who developed a non-functional hand on the paretic side after one year or more of stroke and 25 asymptomatic controls were included. To obtain the CSA of the median nerve at the wrist in the neutral position and the LSL of the median nerve during wrist extension, the participants underwent bilateral ultrasound examination. [Results] The non-paretic side of stroke patients had the largest median nerve CSA, followed by the paretic side and then the controls; the median nerve CSA in the non-paretic side and the controls differed significantly. The CSA of the median nerve was significantly larger in the non-paretic sides of patients, evaluated at more than 5.5 years post-stroke relative to those evaluated at less than 5.5 years. The LSL of the paretic side was significantly shorter than the non-paretic side and the controls. [Conclusion] Compared to the controls, the CSA of the median nerve was enlarged in the non-paretic hand of ambulant chronic stroke patients and the LSL were identical. As such, entrapment neuropathy of the median nerve may follow long-term chronic stroke due to overuse. This study indicates the usefulness of ultrasound in the potential identification of such cases.

Key words: Peripheral nerve, Stroke, Ultrasound
\end{abstract}

(This article was submitted Jul. 22, 2019, and was accepted Sep. 19, 2019)

\section{INTRODUCTION}

Carpal tunnel syndrome (CTS) is a clinical condition resulting from compression of the median nerve where it passes under the transverse carpal ligament in the wrist and is the most common entrapment neuropathy in the upper extremities ${ }^{1)}$. The diagnosis of CTS is often based on clinical examination and is confirmed by electrophysiological studies. Several studies on the ultrasound (US) evaluation of the median nerve have been published. Buchberger et al. were the first to report on the use of US in CTS and since then many studies have reported on the cross-sectional area (CSA) of the median nerve as a diagnostic indication of $\mathrm{CTS}^{2-5}$. The resolution of diagnostic US equipment has greatly improved and it has become possible to use US to evaluate peripheral neuropathies. The CSA at the level of the wrist crease was found to correlate with the sensory nerve conduction velocity $(\mathrm{SNCV})(\mathrm{r}=0.65)$ while the $\mathrm{CSA}$ at the level of the pisiform bone was found to correlate moderately with the SNCV $(\mathrm{r}=0.46)^{6,7)}$.

Based on epidemiological data, CTS is currently considered to be either idiopathic or caused by overuse injury ${ }^{8)}$. Stroke patients are thought to overuse the non-paretic hand and although the non-paretic hand in stroke patients is typically consid-

*Corresponding author. Motoyuki Abe (E-mail: abe-m@seijoh-u.ac.jp)

(C2019 The Society of Physical Therapy Science. Published by IPEC Inc.

(c) (i) $\odot$ This is an open-access article distributed under the terms of the Creative Commons Attribution Non-Commercial No DerivaCC BY NC ND tives (by-nc-nd) License. (CC-BY-NC-ND 4.0: https://creativecommons.org/licenses/by-nc-nd/4.0/) 
ered normal, some studies have reported that CTS on the non-paretic side was a complication of chronic stroke ${ }^{9-11)}$. Sato et al. reported that distal motor latency and SNCV demonstrated greater latency in the non-paretic side in chronic stroke patients ${ }^{11)}$. They also described that sensory disturbance was observed in the median nerve distribution on the non-paretic side in $19.7 \%$ of cases who had a non-functional paretic hand. This has led to speculation that chronic stroke patients are susceptible to CTS. To date, there has only been a single report about US evaluation of the median nerve on both sides in stroke patients, however, this report did not involve a normal control group ${ }^{12)}$.

Previously, US imaging did not allow for the measurement of longitudinal sliding length (LSL) until Dilley et al. developed a software that facilitated this measurement ${ }^{13}$. This method employs a cross-correlation algorithm to determine relative movement between successive frames in a sequence of ultrasound images. Dilley et al. reported the peripheral nerve LSL for the median nerve, the sciatic nerve and the tibial nerve in healthy individuals and individuals with CTS ${ }^{13-16)}$. The LSL of the median nerve in patients with CTS tended to be shorter than that in healthy individuals, however, they did not perform these measurements in stroke patients ${ }^{14}$ ). It remains unclear whether the LSL is altered on both sides in chronic stroke patients when compared with healthy volunteers.

Given the above, we hypothesized that the CSA would be larger on the non-paretic side and that the LSL of the paretic side and the non-paretic side would be shorter than those in healthy individuals. This study aimed to clarify the morphology and longitudinal movement of the median nerve in patients with chronic stroke and compare it with a healthy control group, using US to measure the CSA and LSL on both the paretic and non-paretic sides.

\section{PARTICIPANTS AND METHODS}

Forty patients with stroke were recruited for this study. The types of stroke included 27 cases of intracerebral hemorrhage, 10 cases of cerebral infarctions, and three cases of subarachnoid hemorrhage. The inclusion criteria used were as follows: (1) first-ever stroke, (2) 1 year or more post-stroke, (3) the paretic hand was non-functional, (4) walking independently, including walking with or without crutches or a brace, (5) absence of diabetes mellitus, (6) no medical history of CTS and (7) no severe aphasia. The duration of the period from onset was a median of 65 months (12-176 months). The characteristics of the participants are shown in Table 1. Asymptomatic controls (50 bilateral writs), matched for age, gender, height, and weight were enrolled in this study.

Before US measurements, all participants were interviewed about their present illness and previous history, and the presence of subjective symptoms and numbness in the median nerve area. Participants underwent a physical examination for sensory disturbance, Phalen's maneuver, and Tinel's sign.

B-mode real-time US scanning was performed using a MyLab25 ultrasound machine (Hitachi Medical Corp., Tokyo, Japan) with a 10-MHz linear array transducer. The measurements were performed with the patient in a supine position on a bed, with the elbow extended and the wrist kept in a neutral position. The median nerve was scanned using US in both the transverse and longitudinal planes at the level of the wrist. The landmark of the US measurements was at the level of the pisiform bone of the carpal tunnel in the transverse plane. The CSA was measured using Image J (NIH; https://imagej.nih. gov/ij/).

The longitudinal plane was used to track the fine speckle pattern of selected areas of interest within the nerve and to quantify relative nerve movement based upon the pixel shift that produced the highest correlation coefficient between adjacent frames ${ }^{13}$. The LSL was defined as 60 degrees wrist dorsal extension from the neutral position at the proximal portion of the wrist and was measured using cross-correlation analysis, as previously described ${ }^{13)}$. Furthermore, for the comparison of the CSA and the LSL, the patients were assigned to either a short-term or long-term group based on a median duration of 5.5 years post-stroke.

Table 1. Characteristics of the participants

\begin{tabular}{lccc}
\hline Variables & Stroke patients & Controls & p-value \\
\hline Number of participants (wrists) & $40(80)$ & $25(50)$ & \\
Gender (male/female) & $23 / 17$ & $14 / 11$ & 0.91 \\
Age (years) & $65.0 \pm 9.0$ & $66.3 \pm 8.0$ & 0.65 \\
Height (cm) & $161.0 \pm 8.7$ & $160.4 \pm 18.1$ & 0.81 \\
Weight (kg) & $59.8 \pm 11.9$ & $58.7 \pm 10.9$ & 0.86 \\
Type of stroke (n) & & & \\
Cerebral hemorrhage & 27 & & \\
Cerebral infarction & 10 & & \\
Subarachnoid hemorrhage & 3 & & \\
Duration ( $<5.5$ years/ $\geq 5.5$ years) & $19 / 21$ & & \\
Paretic side (right/left) & $21 / 19$ & &
\end{tabular}


Table 2. The CSA and the LSL in the three groups

\begin{tabular}{lccc}
\hline Parameter & $\begin{array}{c}\text { Control } \\
(50 \text { wrists })\end{array}$ & $\begin{array}{c}\text { Paretic side } \\
(40 \text { wrists })\end{array}$ & $\begin{array}{c}\text { Non-paretic side } \\
(40 \text { wrists })\end{array}$ \\
\hline CSA $\left(\mathrm{mm}^{2}\right)$ & $7.47 \pm 1.87$ & $9.01 \pm 2.65$ & $9.93 \pm 4.22^{*}$ \\
LSL $(\mathrm{mm})$ & $7.08 \pm 1.87$ & $5.75 \pm 1.52^{\#}$ & $6.78 \pm 1.58$ \\
\hline
\end{tabular}

Data are shown as mean $\pm \mathrm{SD}$.

CSA: cross-sectional area; LSL: longitudinal sliding length.

$* \mathrm{p}<0.05$ when compared to control.

${ }^{\#} \mathrm{p}<0.05$ when compared to non-paretic side and control.

Table 3. The CSA and the LSL in the long- and short-term groups

\begin{tabular}{lcc}
\hline Parameter & $\begin{array}{c}\text { Long-term group } \\
(\mathrm{n}=19)\end{array}$ & $\begin{array}{c}\text { Short-term group } \\
(\mathrm{n}=21)\end{array}$ \\
\hline CSA & & \\
$\quad$ Paretic side $\left(\mathrm{mm}^{2}\right)$ & $9.50 \pm 3.32$ & $8.63 \pm 1.91$ \\
$\quad$ Non-paretic side $\left(\mathrm{mm}^{2}\right)$ & $12.11 \pm 5.47^{*}$ & $8.32 \pm 1.64$ \\
LSL & & \\
$\quad$ Paretic side $(\mathrm{mm})$ & $5.79 \pm 1.52$ & $5.71 \pm 1.51$ \\
$\quad$ Non-paretic side $(\mathrm{mm})$ & $6.76 \pm 1.75$ & $6.81 \pm 1.37$ \\
\hline
\end{tabular}

Data are shown as mean $\pm \mathrm{SD},{ }^{*} \mathrm{p}<0.05$ when compared to Short-term group.

CSA: Cross-sectional area; LSL: Longitudinal sliding length.

Long-term group: $\geq 5.5$ years, Short-term group: $<5.5$ years.

There were no significant differences between the paretic side and non-paretic side in both the CSA and LSL measurements.

This study was approved by the Seijoh University Research Ethics Committee (2014C0021) and written informed consent to participate was obtained from all participants.

An unpaired t-test and $\chi^{2}$ test were used to determine the statistical significance of differences between the physical characteristics of the stroke patients and the controls. Data was obtained from three groups: the paretic side, non-paretic side and controls, and the CSA and LSL were analyzed using one-way analysis of variance (one-way ANOVA). Post hoc multiple comparison correction was made using the Bonferroni test. The CSA and LSL in the short-term group and the long-term group were compared using the unpaired t-test. All statistical tests were 2-tailed, with a p-value of less than 0.05 considered to indicate statistical significance. The PASW Statistics ver18 software program was used for this study.

\section{RESULTS}

Four stroke patients (three males and one female) were clinically suspected of having CTS on the non-paretic side based on physical examination. They were all included in the long-term group.

The CSA and the LSL measurements in the three groups are shown in Table 2. The CSA of the median nerve increased in the following order, the non-paretic side, the paretic side, and the controls, with a significant difference between controls and the non-paretic side $(\mathrm{p}=0.012)$. The LSL on the paretic side was significantly shorter compared with the non-paretic side and the controls $(\mathrm{p}=0.002, \mathrm{p}=0.034)$. The CSA and the LSL in the long- and short-term groups were shown in Table3. The CSA of the median nerve on the non-paretic side was significantly larger in the long-term group than in the short-term group $(\mathrm{p}=0.012)$. There was no significant difference in the LSL between the short-term and long-term groups on either side. When comparing the paretic side to the non-paretic side, there were no significant differences in the CSA or LSL.

In the four patients with suspected CTS on the non-paretic side in the long-term group, the CSA was extremely large when compared with the CSA of the controls and the paretic side of the stroke patients, ranging from $12.5 \mathrm{~mm}^{2}$ to $27.2 \mathrm{~mm}^{2}$. The LSL in these same four patients tended to be shorter than those in the control group, ranging from $4.30 \mathrm{~mm}$ to $8.90 \mathrm{~mm}$. Statistical analysis was not performed due to the small number of cases.

\section{DISCUSSION}

In this study, the CSA of the median nerve in the controls was $7.47 \pm 1.87 \mathrm{~mm}^{2}$, which was similar to what has been reported in previous studies ${ }^{2-5)}$. In a report of the CSA of the median nerve in patients with stroke, Uğurlu et al. ${ }^{12)}$ compared the paretic and the non-paretic sides. Uğurlu et al. determined that the CSA was $10.5 \pm 2.0 \mathrm{~mm}^{2}$ on the non-paretic side, 
compared to $9.8 \pm 2.1 \mathrm{~mm}^{2}$ on the paretic side, representing a $7.1 \%$ enlargement on the non-paretic side. In the present study, the CSA was enlarged by $10.2 \%$ on the non-paretic side compared to the paretic side, and was increased by $32.9 \%$ when compared with controls (Table 2). In the study from Uğurlu et al. participants were included with a relatively short disease duration of $14.2 \pm 16$ months and paresis of varying severity. Our study differed from theirs in terms of disease duration and the functional severity of the paretic hand. We therefore assumed that the non-paretic side was more obviously overused and the results of our study seem to support this. Although we did not demonstrate a significant difference in the CSA of the median nerve between the paretic side of the stroke patients and the controls, the value tended to be larger in the paretic side than in the control group. Chronic stroke patients often present with elbow flexion, wrist palmar flexion and finger flexion due to increased muscle tone which causes joint contractures. The change in CSA on the paretic side may therefore be due to abnormal limb positioning and disuse.

It has been reported that US-determined LSL tends to be shorter in patients with CTS ${ }^{14)}$. In our study, the LSL was $7.08 \pm 1.87 \mathrm{~mm}$ in controls (Table 2). A similar LSL value has also been reported in cadavers with wrist dorsal flexion $(7.2 \pm 1.1 \mathrm{~mm})^{17)}$. This shows the methodological reliability of determining LSL by US. The LSL on the paretic side was significantly shorter than the LSL of the controls in both the long- and short-term groups of our study. We postulate that this may be related to the obvious stiffness of the soft tissue on the paretic side. CSA of non-paretic side of the long-term group was significantly larger than the short-term group. The post-stroke duration is considered to influence the morphology of the median nerve owing to overuse, particularly in patients with a non-functional hand. However, there was no significant difference between the LSL of the short-term group and long-term group on the paretic side and the non-paretic side. Thus, the median nerve was restricted in extension on both sides after stroke, even in the short-term, due to stiffness of the soft tissue around the wrist. Dozono et al. reported that excessive use of a walking device was associated with the onset of peripheral neuropathy in the hand on the non-paretic side, based on electrophysiological studies done in chronic stroke patients ${ }^{18)}$. The patients in our study were all ambulant individuals with or without a walking device or brace. With a longer duration after the onset of a stroke, it is possible that CTS will develop in patients who are capable of independently performing ADL's, because the limb on the non-paretic side is used continuously, leading to overuse.

Features suggestive of CTS were observed on the non-paretic side in 4 patients with stroke in the long-term group (10\% of all participants). Notably, the CSA of the median nerve in these patients was extremely large (67.3-264.1\% increase compared to controls). This is likely due to the fact that the median nerve in these individuals was more swollen than in the control group (Fig. 1). Furthermore, the LSL tended to be shorter than in the controls. In light of our findings, similar results from previous US-based studies and findings of the neurological examinations, these patients were suspected of having CTS $^{2-7,13)}$ Many reports of US-examination in CTS have shown that the CSA of the median nerve was enlarged at the level of the inlet of the carpal tunnel ${ }^{5,6}$. This swelling is also noted in individuals with CTS at the level of the pisiform bone, and the CSA of patients with CTS was enlarged by $70-90 \%$ when compared to healthy individuals in previous studies ${ }^{2-5}$ ) Fowler et al. reported that electrophysiological studies of the median nerve in patients with CTS had lower sensitivity and specificity than examination by $\mathrm{US}^{19}$ ) Thus, we consider that US is the preferred means for evaluating suspected cases of CTS, given its ease of use and reliability.

US has become reliable as a diagnostic tool for the assessment of CTS. The change in the CSA that can be detected with US allows early neuropathy evaluation, and US may have more clinical utility than electrophysiological studies. In stroke patients with a non-functional paretic hand with a long period after onset, it is important to evaluate the CSA of the median nerve on the non-paretic side to ensure that function is maintained, even if there are no symptoms. This study was limited in that the relationship between the US findings and the activities of the participant while walking (with or without crutches)

(A)

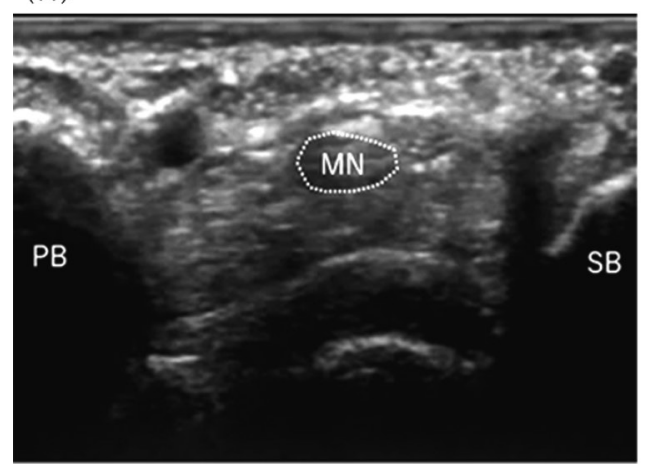

(B)

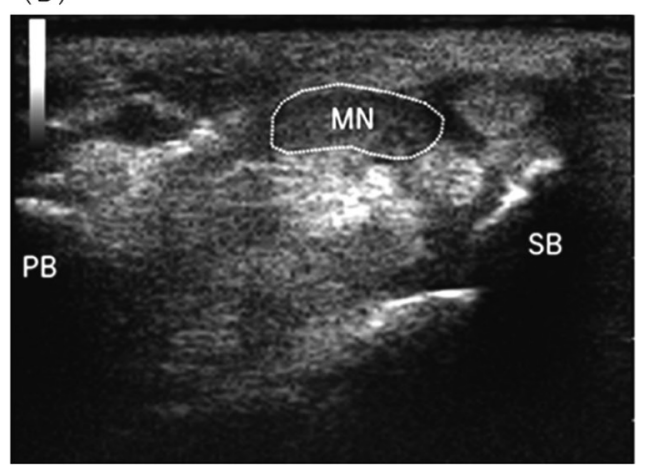

Fig. 1. The cross-sectional area of the median nerve in (A) a control individual, and (B) an individual with suspected carpal tunnel syndrome on the non-paretic side (the sonogram shows the swollen median nerve).

The cross-sectional area of a control individual at the level of the pisiform bone shows the median nerve outlined using ImageJ software. $\mathrm{MN}$ : median nerve; PB: pisiform bone; SB: scaphoid bone. 
were not clarified.

In conclusion, we assessed the CSA and the LSL of the median nerve in patients with chronic stroke and compared it with normal individuals. We revealed that the CSA of the median nerve was the largest on the non-paretic side, particularly in the long-term group. This was deemed to be due to overuse of the non-paretic hand in the patients with stroke, posing a risk of developing CTS. These results indicate that it is important that physicians and therapists pay careful attention to the possibility of CTS in patients with chronic stroke. In future studies, a larger number of patients, including patients with and without independent ambulation, should be investigated.

Funding

No funding was received for this study.

Conflicts of interest

The authors declare no potential conflicts of interest with respect to this research.

\section{ACKNOWLEDGEMENT}

We are extremely grateful to Dr. Andrew Dilley who gave his advice to use the software for measuring the LSL.

\section{REFERENCES}

1) The American Academy of Orthopaedic Surgeon: Clinical practice guideline on the treatment of carpal tunnel syndrome. Rosemont, North River Road, 2008, $1-76$.

2) Buchberger W, Judmaier W, Birbamer G, et al.: Carpal tunnel syndrome: diagnosis with high-resolution sonography. AJR Am J Roentgenol, 1992, 159: 793-798. [Medline] [CrossRef]

3) Duncan I, Sullivan P, Lomas F: Sonography in the diagnosis of carpal tunnel syndrome. AJR Am J Roentgenol, 1999, 173: 681-684. [Medline] [CrossRef]

4) Abe M: Ultrasound measurement of the median nerve for carpal tunnel syndrome of the non-handicapped and the handicapped. J Phys Ther Sci, 2004, 16: 107-114. [CrossRef]

5) Fu T, Cao M, Liu F, et al.: Carpal tunnel syndrome assessment with ultrasonography: value of inlet-to-outlet median nerve area ratio in patients versus healthy volunteers. PLoS One, 2015, 10: e0116777. [Medline] [CrossRef]

6) Nakamichi KI, Tachibana S: Enlarged median nerve in idiopathic carpal tunnel syndrome. Muscle Nerve, 2000, 23: 1713-1718. [Medline] [CrossRef]

7) Mondelli M, Filippou G, Gallo A, et al.: Diagnostic utility of ultrasonography versus nerve conduction studies in mild carpal tunnel syndrome. Arthritis Rheum, 2008, 59: 357-366. [Medline] [CrossRef]

8) Katz RT: Carpal tunnel syndrome: a practical review. Am Fam Physician, 1994, 49: 1371-1379, 1385-1386. [Medline]

9) Kellner WS, Felsenthal G, Anderson JM, et al.: Carpal tunnel syndrome in the nonparetic hands of hemiplegics. Stress-induced by ambulatory assistive devices. Orthop Rev, 1986, 15: 608-611. [Medline]

10) Hunkar R, Balci K: Entrapment neuropathies in chronic stroke patients. J Clin Neurophysiol, 2012, 29: 96-100. [Medline] [CrossRef]

11) Sato Y, Kaji M, Tsuru T, et al.: Carpal tunnel syndrome involving unaffected limbs of stroke patients. Stroke, 1999, 30: 414-418. [Medline] [CrossRef]

12) Uğurlu FG, Tiftik T, Kara M, et al.: Ultrasonographic evaluation of the median and sciatic nerves in hemiplegic patients after stroke. Am J Phys Med Rehabil, 2015, 94: 429-435. [Medline] [CrossRef]

13) Dilley A, Greening J, Lynn B, et al.: The use of cross-correlation analysis between high-frequency ultrasound images to measure longitudinal median nerve movement. Ultrasound Med Biol, 2001, 27: 1211-1218. [Medline] [CrossRef]

14) Erel E, Dilley A, Greening J, et al.: Longitudinal sliding of the median nerve in patients with carpal tunnel syndrome. J Hand Surg [Br], 2003, $28: 439-443$. [Medline] [CrossRef]

15) Ellis R, Hing W, Dilley A, et al.: Reliability of measuring sciatic and tibial nerve movement with diagnostic ultrasound during a neural mobilisation technique. Ultrasound Med Biol, 2008, 34: 1209-1216. [Medline] [CrossRef]

16) Boyd BS, Gray AT, Dilley A, et al.: The pattern of tibial nerve excursion with active ankle dorsiflexion is different in older people with diabetes mellitus. Clin Biomech (Bristol, Avon), 2012, 27: 967-971. [Medline] [CrossRef]

17) Coppieters MW, Alshami AM: Longitudinal excursion and strain in the median nerve during novel nerve gliding exercises for carpal tunnel syndrome. J Orthop Res, 2007, 25: 972-980. [Medline] [CrossRef]

18) Dozono K, Hachisuka A, Wada F, et al.: Peripheral neuropathies in nonparetic upper extremities of stroke patients induced by excessive use of a walking device. J Stroke Cerebrovasc Dis, 2015, 24: 1841-1847. [Medline] [CrossRef]

19) Fowler JR, Cipolli W, Hanson T: A comparison of three diagnostic tests for carpal tunnel syndrome using latent class analysis. J Bone Joint Surg Am, 2015, 97: 1958-1961. [Medline] [CrossRef] 